\title{
Sulle curve razionali di uno spazio lineare ad un numero qualunque di dimensioni
}

\author{
(Di L. Berzolari, a Pavia.)
}

ì noto che $\mathrm{i}$ combinanti del sistema lineare $\boldsymbol{\infty}^{d}$ determinato da $d+1$ forme binarie di ordine $n(n>d)$ coincidono con quelli del sistema lineare $\infty^{n-d-1}$ conjugato (apolare) al primo $\left.{ }^{*}\right)$. In linguaggio geometrico, di qui risulta che, se in uno spazio lineare di $d$ dimensioni si considera una curva razionale $C^{d_{n}}$ di ordine $n$, resta su essa determinata un'involuzione di ordine $n$ e di specie $n-d-1$ (che col sig. STAHL diremo fondamentale), i gruppi della quale sono definiti dalla proprietà di essere apolari a tutte lo sezioni piane della curva, cioè a tutti i gruppi di $n$ suoi punti che sono situati in un piano (**).

Quest'importante involuzione contiene tutte le proprietà della curva, c

(*) Questa propricta, che si estende immediatamente ad un sistema di forme con un numoro qualuncjue di variabili, è stata dimostrata, pressochè nel medesimo témpo, da vari goomotri: Stephanos Gymirssos, Mémoire sur les faisceaux de formes binaires ayant un? mone jacolicme (.rim prós. par dirors savants, cce., Paris, 12 dicembre 1881; tom. 27, pag. 17), e Su la thérie des formes binanes ot sm l'elinimation (Annales scientifiques do 1 École Normale Supérieure, 33." serie, tom. 1, 1884, pag. 23); Bum, Ueber binäre Formen und die Gleichung sechsien Grades (Math. Ann., Bd. 20, pag. 385, 1882); Fraxz Meyse, Apolaritat und rationale Curven, Tübingen, 188\%, pag. 39.

**) In ruesto lavoro denoto con $S_{;}$uno spazio lineare di $i$ dimensioni; in particolaro chiamo piano un $S_{i-1}$ contenuto nello spazio $R_{d}$ di $d$ dimensioni, a cui appartiene $C^{A}{ }_{n}$. Dico inoltre che un $S_{i}$ è osculatore ad una curva in un sto punto, quando esso la in quel funto un contatto d"ordine i colla curva.

Annali di Matematica, tomo XXI. 
può essere stabilita per via più geometrica mediante la teoria delle osculanti (*), come ha mostrato con molti esempi il sig. STAHL (**). Quando sia in particolare $n=d-1$, l'involuzione si riduce ad un unico gruppo, quello formato dai punti di contatto dei $d+1$ piani aventi colla curva un contatto di ordine $d$; ma il sig. Studx (loc. cit.), coll'esempio della $C^{3}$, ha trovato che, dato allora un punto arbitrario nello spazio, resta determinata sulla curva un' involuzione di ordine $n$ o di prima specie $\left.{ }^{*} * *\right)$, la quale fornisce le proprietà più notevoli della curva stessa, e contiene sempre fra' suoi gruppi, qualunque sia il punto preso, il gruppo sopra considerato.

In due reconti lnvori relativi alla $C_{4}^{3}$ ed alla $\left.C_{3}{ }_{3}{ }^{* * * *}\right)$ ho dimostrnto che l'involuzione precedente non è altro che l'involuzione conjugata a quella che si ortiene tngliando la curva coi piani passanti per il punto dato; ed ho inoltre fatto vedere come (sempre nel caso di $n=d+1$ ) tali involuzioni determinate dai punti dello spazio siano intimamente legate con quelle forme, che il sig. Gross (*****) ha chinmato funzioni generatrici indirette dei combinanti elementari relativi alla curva.

Nel presente lavoro mi oceupo di una $C^{d}{ }_{n}$ qualunque, e in primo luogo, dopo aver accennato al significato geometrico delle funzioni generatrici dirette, espongo brevemente come, partendo, al modo del sig. Stafr, dalla considerazione delle osculanti, si possa costruire l'involuzione fondamentale; dimostro inoltre che questa possiede in generale un gruppo piano (ed uno solo) nell'unico caso in cui sia ad un tempo $d$ pari ed $n$ dispari. In seguito determino il significato geometrico di tutte le funzioni generatrici indirette, dimostrando alcuni notevoli teoremi sulle osculanti normali, cioè di ordine $d$; poscia, ge-

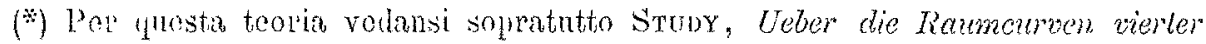

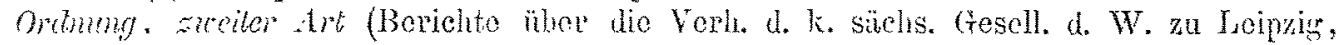
11 remajo 1880); Jotus, Die Theorie der Osculanten, cec. Aachon, 1886, ed inoltro i ]itropi lel sir. Stum c mici che citerò or ora.

(w) Cli interessanti laromi del sig. Sturt su questo argomento si trowano nei volami 101 e 104 del cropnale di CruLLF, e trattano delle curve piane degli ordini 3,4 o 5 , * lalle curre golblo legli ordini $1,5,6$ e 7 .

(*) Un caso particolare di tali involnzioni rolative alla $C^{3}$ (quella determinata dal

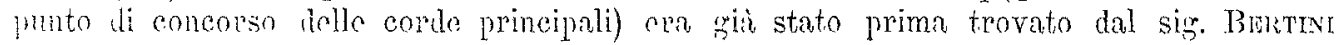
(Rondic. thel R. Istituto Lombardo, 20 gingno 1872).

(*) Sui combinanti dei sistemi di fome binarie, cee. (Ammali di Matem., serie II,

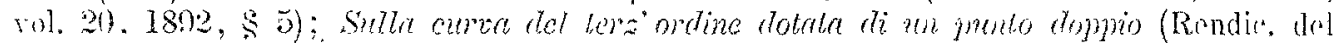
li. Istituto Lomhar: 14 lnglio 1802$)$.

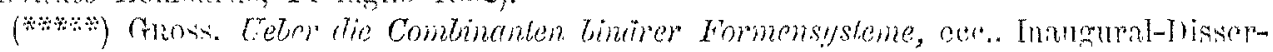

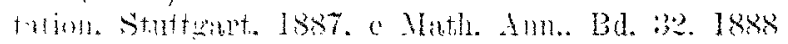


neralizzando i ricordati risultati contenuti nei lavori del sig. STudy e miei (relativi ai casi di $h=1$, e $d=2, n=3 ; d=3, n=4$ ), trovo che in generale, dato un $S_{h-1}$ qualunque ( $n \geqslant d \geqslant h \geqslant 1$ ), viene determinata sulla curvn un' involuzione $\left[S_{n-1}\right]_{n-d+h-1}^{n}$ di ordine $n$ e di specie $n-d+h-1$, la quale, qualunque sia l' $S_{h-1}$ da cui si parte, contiene fra' suoi gruppi tutti quelli dell'involuzione fondamentale. L'involuzione $\left[S_{k-1}\right]^{n} n_{n-a+k-1}$, a cui si perviene geometricamente colla considerazione delle osculanti, e di cui ho pure assegnata un'altra costruzione molto semplice, non è che l'involuzione conjugata a quella che s'ottiene tagliando la curva coi piani passanti per il dato $S_{l-1}$. Uguagliando a zero l' $(h+1)^{\text {ma }}$ funzione generatrice indiretta, in cui si siano sostituite le coordinate dell' $S_{k-1}$, si ottiene appunto l'equazione della detta involuzione, cioè la relazione cui devono soddisfare $n-d+h$ punti appartenenti ad uno stesso gruppo della medesima. Se invece si suppongono dati valori arbitrari ai parametri di quegli $n-d+h$ punti, la stessa equazione rappresenta, in coordinate di $S_{h-1}$, un certo $S_{d-h}$, determinato dalle osculanti normali di quei punti, presi ad $n-d+1$ per volta.

Da ultimo determino i gruppi neutri dell'involuzione fondamentale nonchì delle involuzioni $\left[S_{h-1}\right]_{n-d+h-1}^{n}$, e stabilisco alcune proprietà dei gruppi piani di queste ultime: proprietà che sono differenti secondo che tanto $d$ quanto $a k$ sono pari od impari.

Parechi dei risultati precedenti si possono stabilire per via puramente geometrica, considerando (come ho fatto specialmente nei n.i 5,6 e 12) la curva $C_{n}^{n}$ come projezione di una curva normale di ordine $n$ di uno spazio ad $n$ dimensioni: con questo metodo tutie le involuzioni, di cui sopra si è discorso, derivano da alcune semplicissime e ben note proprietà dell'ultima curva.

1. In uno spazio lineare $R_{d}$ a $d$ dimensioni le coordinate omogenee del punto corrente $\lambda$ della curva $C^{d l_{n}}$ siano espresse da

$$
\begin{gathered}
\rho x_{i}=f_{i}(\lambda)=a_{i 0} \lambda^{n}+\cdots+a_{i n} \\
(i=1,2, \ldots, d+1),
\end{gathered}
$$

e si abbia simbolicamente

$$
f_{i}(\lambda)=a_{i, \lambda}^{n_{2}, \lambda}=\left(a_{i_{2},} \lambda_{1}+a_{i_{2},} \lambda_{2}\right)^{n}
$$

La curva possiede (Gross, loe. cit.) $d+1$ funzioni generatrici dirette $G^{\prime}, G_{1}^{\prime}, \ldots, G_{d-1}^{\prime}, G_{d}$, una qualunque delle quali, per es. la $(v+1)^{\text {ma }}$, si 
deduce dal detcrminantc

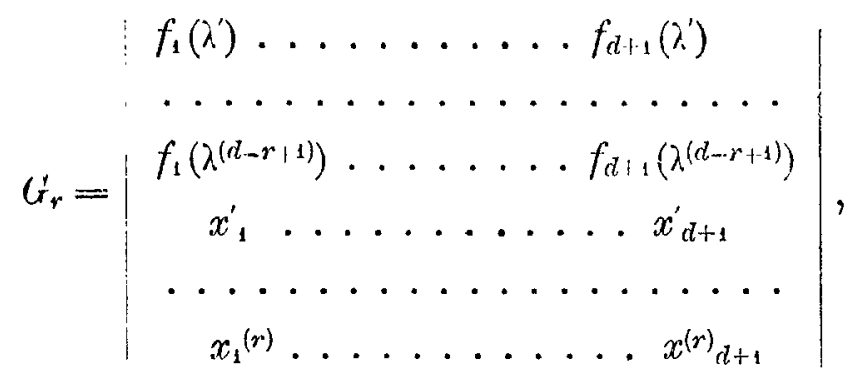

dividendolo per $\mathrm{i}$ determinanti $\left(\lambda^{\prime} \lambda^{\prime \prime}\right) \ldots$ Da ciò risulta senz'altro:

L'equazione $G^{\prime}=0$ dà la condizione perchè $i d+1$ punti della curva, dei quali nell' equazione stessa figurano i parametri $\lambda^{\prime}, \ldots, \lambda^{\left(d^{+-1}\right)}$, siano in uno stesso piano.

L'equazione

$$
G^{\prime}=0 \quad(r=1,2, \ldots, d)
$$

rappresenta, in coordinate di $S_{r-1}\left(^{*}\right), l$ $S_{d-r}$ determinato dai $d-r+1$ punli della curva, di cui nell' equazione stessa compajono $i$ parametri, ciò̀ $\dot{e}$ soddisfatta dalle coordinate locali di tutti gli $S_{r-1}$ aventi ciascuno un punto comune coll' $S_{d-r}$ considerato.

In particolare, l'equazione $G_{d}=0$ rappresenta, in coordinate di piani, il punto $\lambda$ della curva.

Rappresentando simbolicamente con $\alpha^{2 x} \lambda$ il primo membro, o chiamando $u_{1}, \ldots, u_{d+1}$ le coordinate omogenee di un piano, si avrà quindi

$$
G_{d}=\alpha_{\lambda}^{n_{\lambda}}=\sum_{1}^{d+1} u_{i} f_{i}(\lambda)
$$

2. La curva $C_{n}{ }_{n}$ ha pure (Gross, loc. cit.) $d+1$ funzioni generatrici indirette $\Gamma^{\prime}, \Gamma_{1}^{\prime}, \ldots, \Gamma_{d}^{\prime}$, e, volendo trovarne l'interpretazione geometrica, cominciamo a considerare la $\Gamma^{\prime}$, richiamandone il modo di generazione. Siano $\varphi_{1}, \ldots, \varphi_{n-d}$ le forme binarie di grado $n$ apolari alle $d+1$ forme $f_{i}$, e colle medesime si formi il determinante

$$
\begin{gathered}
\varphi_{1}\left(\lambda^{\prime}\right) \ldots \ldots \ldots \ldots \varphi_{n-d}\left(\lambda^{\prime}\right) \\
\ldots \ldots \ldots \ldots \ldots \ldots \\
\varphi_{1}\left(\lambda^{(n-d)}\right) \ldots \ldots \ldots \varphi_{n-d}\left(\lambda^{(n-d)}\right)
\end{gathered}
$$

(*) Cirea lo coordinate di un $S_{i}$ o le relazioni a cui esse soddisfamo, vodasi J'Ovnn, Le funsioni melviche fondamentali negli spazi di quanle si vogliano dimensioni e clitvalura costanle (Mem. della R. Lecal. dei Lincei, serie III, vol. 1, 1877). 
Dividendolo per $\mathrm{i}$ determinanti $\left(\lambda^{\prime} \lambda^{\prime \prime}\right), \ldots$, e rappresentando il quoziente come funzione dei coefficienti delle $f_{i}$ (ciò che è possibile in virtù delle relazioni bilineari che, per l'apolarità, hanno luogo fra i coefficienti delle $f_{i}$ o quelli delle $\varphi_{k}$ ), si ottiene la funzione che si è chiamata $\Gamma^{\prime}$. Ora il sistema lineare determinato dalle forme $\varphi_{k}$ rappresenta manifestamente l'involuzione fondamentale, e l'equazione $\Gamma^{\prime}=0$ dà, com'è noto, la condizione perchè i parametri $\lambda^{\prime}, \ldots, \lambda^{(n-l)}$ siano radici di una stessa forma di quel sistema lineare, laonde:

L'equazione $\Gamma^{\prime}=0$ esprime la condizione perchè gli $n-d$ punti dellu curva, di cui essa contiene $i$ parametri, appartengano ad uno stesso gruppo dell' involuzione fondamentale.

3. In ciò che segue rappresenteremo col simbolo $\left[\lambda, \ldots, \lambda^{(n-x)}\right]_{r}$ l'oseulante mista dei punti $\lambda^{\prime}, \ldots, \lambda^{(n-r)}$ della curva, ossia la curva razionale d'ordine $r$, il cui punto corrente $\lambda$ ha per equazione

$$
\alpha_{\lambda}, \cdots \alpha_{\lambda}(n-n) \alpha_{\lambda}^{r}=0
$$

Consideriamo ora l'osculante $\left[\lambda^{\prime}, \ldots, \lambda^{n-a^{2}-1}\right] d+4$ è noto $\left(^{*}\right)$, o si dimostra del resto assai facilmente, che essa contiene $d+1$ punti stazionar $i$, ciod tali che per ciascuno di essi esiste un piano (stazionario) avente ivi colla curva un contatto di ordine $d$; si trova subito che i parametri di tali punti sono dati dall' equazione

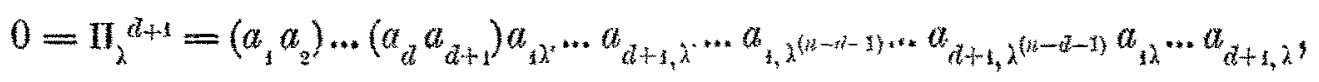

laonde questi punti e gli $n-d-1$ punti dati sono rappresentati complessivamente dalla seguente equazione in $\lambda$ :

$$
\left(\lambda \lambda^{\prime}\right) \ldots\left(\lambda \lambda^{\left(3-2 d^{2}-1\right)}\right) \Pi_{\lambda}^{d+1}=0
$$

Ora il primo membro è apolare a ciascuna delle forme $f_{i}$, epperò:

Dati $n-d-1$ punti qualunque sopra $C^{\prime \prime} n, i d+1$ punti che con essi costituiscono un gruppo dell' involuzione fondamentale lanno per parametri quelli dei punti stazionari dell osculante mista di ordine $d+1$ determinata dai punti considerati.

4. Indicando con $\lambda, \ldots, \lambda^{(n)}$ i parametri di $n$ punti di $C_{n}^{d}$ formanti uu gruppo dell' involuzione fondamentale, avremo identicamente, qualunque

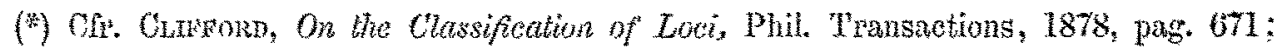
Lonu, Intorno alle curve rasionali $l^{\prime}$ ortine is dello spasio a $n-1$ dinension (Rend. de1 Circolo Vat di Palermo, tom. 2, 1888, jng. 20:3). 
siano le $u_{i}$,

$$
\alpha_{\lambda^{\prime}} \ldots \alpha_{\lambda^{\prime \prime \prime}}=0 \text {. }
$$

Se poi $u^{\prime}$ ̀̀ il parametro di uno dei punti stazionari dell'osculante $[\lambda, \ldots$, $\left.\lambda^{(n-\ell-1)}\right]_{d+1}$, l'osculante normale $\left[\lambda^{\prime}, \ldots, \lambda^{(n-l-1)}, \mu^{\prime}\right]_{d}$ sarà contenuta interamente nel relativo piano stazionario. Ora il punto corrente $\lambda$ di questa curva lia per equazione

$$
\alpha_{\lambda}, \ldots \alpha_{\lambda}(1+-1-1) \alpha_{p^{\prime}} \alpha_{\lambda}^{d}=0
$$

quindi, se nel primo membro in luogo delle $u_{i}$ si sostituiscono le coordinate del detto piano stazionario, esso si annulla qualunque sia $\lambda . \mathrm{Si}$ ottengono in tal modo le equazioni seguenti:

$$
\begin{gathered}
\alpha_{\lambda} \ldots \alpha_{\lambda}(1+\lambda-1) \alpha_{\mu} \alpha_{1}{ }^{r} \alpha_{2}^{d l-r}=0 \\
(r=0,1, \ldots, d),
\end{gathered}
$$

lo quali, in virtù dell'identità (1), equivalgono soltanto a $d$, e possono servire a determinare le coordinate del piano stazionario considerato. La simmetria delle (2) rispetto ai parametri $\lambda^{\prime}, \ldots, \lambda^{(n-l l-1)}, \mu^{\prime}$ fornisce il teorema seguente:

Sia dato un gruppo qualunque dell' involuzione fondamentale, e di esso si considerino $n-d$ punti qualunque: questi, presi ad $n-d-1$ per volta, determinano $n-d$ osculanti miste d'ordine $d+1$, le quali hanno tutte un. piano stazionario comune; esso tocca ciascuna nel punto avente per parametro quello del punto rimanente.

Chiamando $u^{\prime \prime}, \ldots, \mu^{(d+1)}$ gli altri punti della curva che completano i] gruppo sopra considerato dell'involuzione fondamentale, dalle (2) seguono successivamente altri $d-1$ gruppi di equazioni, di cui uno qualunque 2 il soguente:

$$
\begin{gathered}
\alpha_{\lambda^{\prime}} \ldots \alpha_{\lambda}(m-1,-1) \alpha_{k^{\prime}} \ldots \alpha_{\mu^{(k)}} \alpha_{1}{ }^{\prime \prime} \alpha_{2}^{d-r^{\prime}-k_{+1}}=0 \\
(r=0,1, \ldots, d-k+1) .
\end{gathered}
$$

Le sue $d-k+2$ equazioni, in virtù della (1), equivalgono a sole $a-l i+1$, e dicono che, se di un gruppo dell' involuzione fondamentale si considerano $n-d+k-1$ punti qualunque, essi, presi ad $n-d$ per volta, determinano ogni volta un piano nel modo indicato dal tenrema precedente: questi $\left(\begin{array}{c}n-d+k-1 \\ n-d\end{array}\right)$ piani passano tutti per un medesimo $S_{d-k}$, quello appunto che è rappresentato dall'ultimo gruppo di equazioni. 
Raccogliendo, si può enunciare la proprietà seguente:

Dati $n$ punti della curva costituenti un gruppo qualunque dell'involuzione fondamentale, essi, presi ad $n-d$ per volta, determinano ogni volta un piano, nel modo sopra indicato: gli $\left(\begin{array}{l}n \\ d\end{array}\right)$ piani che cosi in tutto si ottengono sono le facce di un ennagono completo.

I vertici di questo ennagono si possono coordinare ad uno ad uno ai punti del gruppo considerato, intendendo che ad ogni vertice venga coordinato quello fra $\mathrm{i}$ punti del gruppo, che, unito con altri $n-d-2$ punti qualunque del gruppo stesso, non dà mai luogo ad un'osculante di ordine $d+1$, di cui un piano stazionario passi per il vertice considerato (*).

5. Ai risultati precedenti si può giungere anche colla pura geometria, immaginando (**) che la $C^{d}{ }_{n}$ sia ottenuta projettando in $R_{d}$ da uno spazio lineare ad $n-d-1$ dimensioni (che in tutto il seguito terremo fisso e denoteremo con $\Sigma_{n-d-1}$ ) una curva normale $C_{n}^{n}$ di ordine $n$ di uno spazio lineare $R_{n}$ ad $n$ dimensioni passante per $R_{d}$ e per $\Sigma_{n-d-1}$. Secondo che $n$ è pari od impari, la $C^{n}$ determina (***) una polarita rispetto ad una quadrica $F$ passante per la curva, oppure un sistema nullo $\Theta$, e se diciamo $\Sigma_{a}$ lo spazio che per tal modo è conjugato a $\Sigma_{n-d-1}$, le involuzioni che si ottengono tagliando $C_{n}$ cogli $S_{n-1}$ passanti per $\Sigma_{n-t-1}$ e per $\Sigma_{t l}$ sono fra loro conjugate (Cfr. Casternuovo, loc. cit., § 2.).

Di qui risulta che l'involuzione fondamentale sopra $C^{d}{ }_{n}$ si può ottenere tagliando $C_{n}^{n}$ cogli $S_{n-1}$ passanti per $\Sigma_{l l}$ e projettando le intersezioni $d a$ $\Sigma_{n-l l-1}$ sopra $R_{l l}$.

Un'osculante qualunque $\left[\lambda^{\prime}, \ldots, \lambda^{(v)}\right]_{n-r}$ di $C^{n}{ }_{n}$ ̀े una curva normale situata in un $S_{n-r}$, il quale è lo spazio comune agli $S_{n-1}$ osculatori a $C_{n}^{n}$ nei punti $\lambda, \ldots, \lambda^{(r)}$ : projettando questa curva da $\Sigma_{n-l-1}$ su $R_{d l}$, si ottiene l'osculante $[\lambda, \ldots, \lambda(r)]_{n \rightarrow r}$ di $C_{n}^{\prime}$. Presi allora $n-\lambda$ punti di $C_{n n}^{n}$ situati in un $S_{n \rightarrow 1}$ passante per $\Sigma_{d}$, gli $S_{n-1}$ che osculano $C_{n}^{n}$ in quei punti si tagliano in un $S_{d}$ avente un punto comune col $\Sigma_{n-i-1}$; la projezione di tale $S_{d}^{\prime}$ da $\Sigma_{n-d-1}$

(*) Cir. STAm, Uabo dio Findamentrimolutionen auf rationalon Curon (Giomale di Creirf, Bu. 104, 1888).

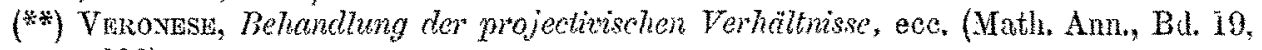
1881, pag. 208 ).

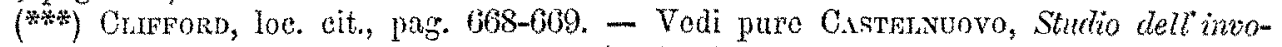
inzione generale sulle mure razionali, ecc. (Atti lel R. Istituto Veneto, fom. A, serie VI. 1880$, s 1$)$. 
dà quindi in $R_{d}$ un $S_{l-1}$, che manifestamente è un piano stazionario per tatte le osculanti di ordine $d+1$ determinate dalle projezioni degli $n-d$ punti lati, presi ad $n-d-1$ per volta.

Presi poi sopra $C_{n}^{d} n$ punti costituenti un gruppo dell' involuzione fonlamentale, i punti corrispondenti di $C_{n z}^{n}$ saranno $\mathrm{i}$ punti di contatto degli $S_{n-1}$ osculatori a $C_{n}^{n}$, uscenti da un determinato punto $A$ di $\Sigma_{n-l l-1}$. Gli $S_{i}$ che questi $S_{n-s}$, considerati ad $n-1$ per volta, hanno in comune, formano un $n$-spigolo completo avente il vertice nel punto $A$, e, projettandone gli $n$ spigoli da $\Sigma_{n-7-1}$ su $R_{d}$, si hanno i vertici dell'ennagono completo di cui si parla nel numero precedente. I teoremi ivi contenuti sono dunque dimostrati.

6. Cerchiamo se fra i gruppi dell'involuzione fondamentale qualcuno abbia tutti i suoi punti in un piano. Perchè ciò possa accadere è intanto necossario che $n$ sia dispari; in tale ipotesi sia

$$
\underbrace{+1}_{i} u_{i} x_{i}=0
$$

l'equazione di un piano: esso taglia $C_{n}^{d}$ nei punti i eni parametri sono le radici dell' equazione

$$
\sum_{i}^{l+1} u_{i} \alpha^{n} i_{i, \lambda}=0
$$

Volendo determinare le $u_{i}$ in modo che $j$ primo membro sia apolare a tntte le sezioni piane di $C_{n}$, scriviamo dapprima le condizioni perchè esso sia apolare alle sezioni fatte coi piani fondamentali $x_{1}=0, \ldots, x_{l}=0$, ossia a cinscuna delle forme $a^{n}{ }_{1,2}, \ldots, a^{n}{ }_{l, \lambda}$. Ponendo

di guisa che sarà

$$
A_{r s}=\left(a_{r} a_{s}\right)^{n}
$$

$$
A_{r s}=-A_{s r} \text {, }
$$

ed oliminando lo $u_{i}$ dalla (3) e dalle $d$ equazioni di condizione, si ottieno:

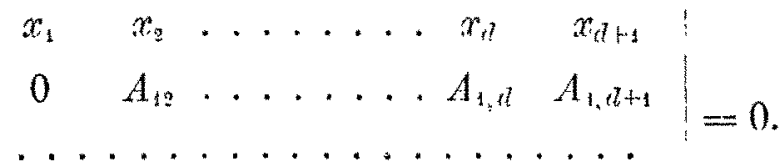

$$
\begin{aligned}
& A_{l, 1} \quad A_{t, 2} \ldots \ldots \ldots, 0 \quad A_{l l, t i 1}
\end{aligned}
$$

Ora se $d$ o impari, il coefficiente di $r_{d_{1}}$ in quest'equazione è un determinante gobbo simmetrico di ordine impari nd quindi nullo: in tal caso 
pertanto il piano rappresentato dalla (4) passa per il punto $x_{1}=\cdots=x_{l l}=0$ e nell'involuzione fondamentale non esiste alcun gruppo piano. Se al contrario d è pari, il piano (4) non passa, in generale, per il punto ora nominato, quindi la sezione fatta nella curva da quel piano, essendo apolare a $d+1$ sezioni piane linearmente indipendenti, è apolare a tutte. Si conclude dunque:

L'involuzione fondamentale possiede in generale un gruppo piano (ed uno soltanto) nell' unico caso in cui sia ad un tempo d pari ed n impari.

Alla stessa conolusione si perviene immediatamente riprendendo le considerazioni del numero precedente. Dato un $S_{n-1}$ passante per $\Sigma_{d}$, gli $n$ punti in cul esso taglia $C_{n}^{n}$ verranno projettati da $\Sigma_{n-d-1}$ in $n$ punti di $C_{n} l_{n}$ situati in un piano, soltanto quando l' $S_{n-1}$ considerato passi per $\Sigma_{n-d-1}$, cioè quando gli spazi $\Sigma_{n-l-1}$ e $\Sigma_{d}$ si taglino. Ora, per note proprietà, ciò non può accadere se $n$ ̀̀ pari, poichè in tal caso quei due spazi sono conjugati rispetto alla quadrica $F\left({ }^{*}\right)$; quando $n$ invece sia impari, quegli spazi sono conjugati nel sistema nullo $\theta$, epperò si tagliano, necessariamente, nel solo caso in cui l'uno, e quindi anche l'altro, sia ad un numero pari di dimensioni, quando cioè $d$ sia pari.

7. Sulla curva $C_{n}^{t}$ si considerino $n-d+1$ punti qualunque $\lambda, \ldots$, $i^{(n-l+1)}$, i quali, presi ad $n-d$ per volta, determinano $n-d+1$ osculanti normali. Fissandone una, per es. la $\left[\lambda^{\prime}, \ldots, \lambda^{(n-l l)}\right]_{d}$, le coordinate del suo punto corrente $\lambda$ sono

$$
\begin{gathered}
x_{i}=a_{i, \lambda} \ldots a_{\left.i, \lambda^{(j)}, a^{\prime}\right)} a_{i, \lambda}^{d} \\
(i=1,2, \ldots, d+1),
\end{gathered}
$$

o però le coordinate del suo piano oseulatore nel punto avente per parametro $\lambda^{\left(n \cdot t^{\prime}\right)}$ (cioè quello del punto che si è trascurato) sono

$$
u_{1} \equiv\left(a_{2}^{3} a_{3}\right) \ldots\left(a_{a l} a_{l l+1}\right) a_{2, \lambda,} \ldots a_{d+1, \lambda} \ldots a_{2, \lambda}(u-d+1) \ldots a_{d+1, \lambda} \lambda^{(n-a+1)},
$$

- Ia loro simmetria rispetto a $\lambda^{\prime}, \ldots, \lambda^{(n-m+1)}$ dimostra il teorema:

Dati $n-d+1$ punti qualunque sopra $C^{d}{ }_{n}$, essi, presi ad $n-d p^{r}$

(*) Cirua le ruadricho di uno spazio lineapo qualunque vedansi Vurovess, loc, eit.,

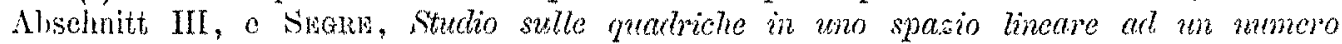

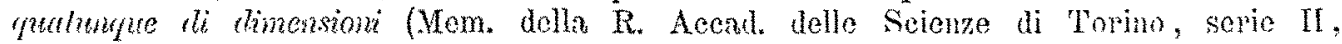
tom. $3(;, 1881)$.

Annali di Malematioa, tomo XXI. 
volta, determinano $n-d+1$ osculanti normali, le quali hanno un piano osculatore comune; questo oscula ciascuna nel punto che ha per parametro quello del punto rimanente.

E altresi da notare che in tal piano tutte le osculanti ora nominate hanno pure in comune l'osculante $\left[\lambda^{\prime}, \ldots, \lambda^{(n-d+1)}\right]_{d-1}$, che è una curva normale del piano stesso.

L'equazione del piano suddetto si ottiene uguagliando a zero la funzione generatrice indiretta $\Gamma_{1}^{\prime}$, formata coi parametri $\lambda^{\prime}, \ldots, \lambda^{(n-d+1)}$ dei punti sopra considerati.

Per convincersi di ciò basta tener presente il modo con cui si forma la funzione $\Gamma_{t}^{\prime}$, e far uso di un teorema generale dovuto al sig. Stempranos (Mimoire sur les faisceaux, ecc., loc. cit., pag. 22); ma su questo non mi fermo più oltre, poichè avrò occasione di ritornarvi nel $n .^{\circ} 11$ per altro fine.

I ragionamenti fatti sopra si possono proseguire, considerando sulla curva $n-d+2, n-d+3, \ldots$ punti qualunque, e si perviene cos̀ al teorema generale:

Dati $n-d+h$ punti qualunque sopra $C_{n}^{d}(n \geqslant d \equiv h \geqslant 1)$, prendendoli ad $n-d+1$ per volta, essi determinano ogni volta un piano, nel modo indicato poc'anzi: gli $\left(\begin{array}{c}n-d+h \\ h-1\end{array}\right)$ piani che cosi si ottengono passano tutti per uno stesso $S_{l-h}$; essi sono inoltre le facce di un moltispazio completo costituito di $n-d+h$ spazi $S_{d-h+1}$ ed avente per sostegno l' $S_{d-h}$, ciò̀ sono $i$ piani che contengono, in tutti $i$ modi possibili, $h-1$ di questi $S_{d-h+1}$.

Gli $S_{c l-h+1}$ del moltispazio precedente si possono coordinare ad uno ad uno agli $n-d+h$ punti dati di $C^{l} n$, intendendo che ad ognuno degli $S_{l-h+1}$ si faccia corrispondere quello fra $i$ punti dati, che, unito con altri $n-d$ qualunque, non determina mai, nel modo sopra esposto, un piano passante per l' $S_{t-h+1}$ considerato.

L'equazione che risulta uguagliando a zero $l(h+1)^{\mathrm{ma}}$ funzione generatrice indivetta $\Gamma^{\prime}{ }_{h}$, nella quale si siano sostituiti $i$ parametri degli $n-d+h$ punti dati, rappresenta, in coordinate di $S_{h-1}$, lo spazio nominato $S_{d-h}$, ciò̀ $\grave{e}$ soddisfatta dalle coordinate di tutti gli $S_{h-1}$ aventi un punto comune coll' $S_{l-h-h}$.

La prima parte si può anche enunciare dicendo:

Dati sulla curva $n-d+h$ punti qualunque, considerandoli ad $n-d+$ $h-1$ per volta, essi determinano $n-d+h$ osculanti miste di ordine $d-h+1$, le quali posseggono un $S_{d-h}$ osculatore comune; questo oscula ciascuna nel punto avente per parametro quello del punto trascurato. 
Lo spazio $S_{d-h}$ verrà detto coordinato al gruppo degli $n-d+h$ punti dati sopra $C_{n}^{d_{n}}$.

8. Le equazioni dell' $S_{d-h}$ si possono stabilire nel modo seguente, da cui si ricavano ulteriori conseguenze. Fissati ancora sulla curva $n-d+h$ punti qualunque $\lambda^{\prime}, \ldots, \lambda^{(n-a t+h)}$, si consideri l'equazione

$$
\alpha_{k}, \ldots \alpha_{i}(t-a+h) \alpha_{j}^{d-h}=0 \text {, }
$$

che per chiarezza scriverò cos̀̀:

$$
\alpha_{\lambda} \cdots \alpha_{\lambda}(i n-d) \alpha_{2}(n-d+1) \cdots \alpha_{2}(b-a+h) \alpha_{\lambda}^{d-h}=0 .
$$

Poichè

$$
\alpha_{\lambda} \ldots \alpha_{\lambda}(1 t-n) \alpha_{\lambda}^{d}=0
$$

rappresenta il punto $\lambda$ corrente sull osculante normale $\left[\lambda^{*}, \ldots, \lambda^{(n-c)}\right]_{d}$, è chiaro che, dati i parametri $\lambda^{\prime}, \ldots, \lambda^{(n-a t+h)}$ e $\lambda$, la (5) rappresenta il punto comune allo spazio $S_{h}$ osculatore alla detta osculante nel punto $\lambda$, ed ai piani osculatori alla medesima nei punti $\lambda^{(n-d+1)}, \ldots, \lambda^{(n-d+h)}$ (piani che hanno in comune un $S_{d-h}$ ). L'equazione (5) sarà soddisfatta, in particolare, dalle coordinate di questi $h$ piani, e ciò per qualunque valore di $\lambda$. Avranno dunque luogo, per le coordinate dei piani stessi, le identità

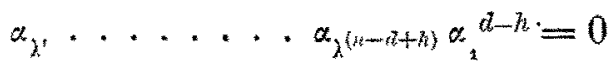

$$
\begin{aligned}
& \alpha_{\lambda_{1}} \ldots \ldots \ldots \alpha_{\lambda^{(H+l t+h)}} \alpha_{1}^{d-l l-1} \alpha_{2}^{3}=0 \\
& \ldots \ldots \ldots \ldots \ldots \\
& \alpha_{\lambda^{\prime}} \ldots \ldots \alpha_{\lambda^{(t+2+\alpha}(+h) \alpha_{2}}^{d-h}=0 \text {. }
\end{aligned}
$$

La simmetria delle medesime rispetto a tutti i parametri $\lambda$ prova che $i$ piani, che si ottengono nel modo ora esposto eseguendo tutte le possibili permutazioni delle $\lambda$, passano tutti per i $d-h+1$ punti rappresentati dalle (6), e quindi per uno stesso $S_{a-h}$, ciò̀ quello determinato dai punti stessi.

9. I piani precedenti sono in numero di $\left(\begin{array}{c}n-h+h \\ h-1\end{array}\right)$, e poichè per $n \equiv d$ questo numero non è minore di $h$, lo spazio $S_{l l-h}$ comune ai piani stessi viene certamente da questi determinato. Ora le (6) si possono compendiare dicendo che, quando per le $u_{i}$ si sostituiscano le coordinate di uno qualunque di quei piani, riesce identicamente nulla, qualunque sia $\lambda$, la forma

$$
\alpha_{2}, \ldots \alpha_{2}(n-i+k) \alpha_{\lambda}^{d-k},
$$


ossia dicendo che il gruppo dei punti presi $\lambda^{\prime}, \ldots, \lambda^{(\eta-n+l)+h)}$ è apolare al gruppo di $n$ punti che si ha secando $C^{\prime b}$ con uno qualunque di quei piani, epperò, per l'osservazione premessa, con uno qualunque dei piani passanti per l' $S_{t l-\eta_{t}}$ determinato dai piani stessi. Pertanto possiamo dire:

Dati $n-d+h$ punti qualunque di $C_{n}{ }_{n}(n \geqslant d \geqslant h \geqslant 1)$, il gruppo da cssi custituito $i$ apolave a tutti $i$ gruppi di $n$ punti che si otlengono tagliando la curva coi piani passanti per l' $S_{l-h}$ coordinato al gruppo dato di punti.

10. Dato ad arbitrio un $S_{h-1}$, esistono $\boldsymbol{\infty}^{h_{-1}-l_{+} h-1}$ gruppi di $n-d+h$ punti della curva, il cui $S_{t-h}$ coordinato abbia un punto comune coll' $S_{h-1}$. Invero se in

$$
\Gamma_{k}=0
$$

supponiamo sostituite le coordinate del dato $S_{h-1}$, otteniamo an'equazione di grado $d-h+1$ rispetto a ciascuno dei parametri $\lambda^{\prime}, \ldots, \lambda^{(n-7 \cdot 1)}$, di modo che, assegnati valori arbitrari ad $n-d+h-1$ qualunque di essi, restano determinati $d-h+1$ valori del rimanente: la proprietà enunciata ha allora luogo in virtù di quanto si è detto alla fine del n. ${ }^{\circ}$. Si ha pertanto il teoroma generale:

Fissato in $R_{l}$ uno spazio lineare qualunque $S_{h-1}$, nesta determinata sopra $C_{n}^{t}$ wh' involuzione $\left[S_{h-1}\right]_{n-a+k-1}^{n}$ di or dine ne di specie $n-d+h-1$, un grupro qualtunque della quale gode della propriet⿳亠口冋 che lo spazio $S_{t b-n}$, coordinato ad un gruppo arbitrario di $n-d+h$ fra' suoi punti, ha un punto comune col dato $S_{k-1}$. L'equazione di tale involuzione, ossia la relazione che intercede fra $n-d+\hbar$ punti appartenenti ad un suo gruppo, si ottiene uguagliando a zero la funzione $\Gamma_{h}$, nella quale si siano sostituite lo coordinate del duto $S_{h-1}$.

Si può inoltre dimostrare che l'involuzione stessa è conjugata a quella che risulta tagliando $C_{n}^{\text {al }}$ coi piani passanti per il dato $S_{h-1}$; dal che segue cho, qualunque sia questo $S_{h-1}$, essa contiene fra' suoi gruppi intti quelli dell' involuzione fondamentale.

Invero, considerando un gruppo qualunque della detta involuzione e scegliendo $n-d+h$ de'suoi punti, ad essi è coordinato un $S_{d-h}$ avente un punto comune col dato $S_{h-1}$, e giacente quindi con questo in un piano determinato. Tale piano, per il teorema del numero precedente, taglia $C_{n}{ }_{n}$ in $n$ punti costituenti un gmppo apolare a quello degli $n-d+h$ punti considerati, e percio apolare anche all'intiero gruppo fissato dell'involuzione. Quest' ultimo è pertanto apolare a ciasouno degli $(a-n)$ gruppi di $n$ puntí de- 
terminati, nel modo ora esposto, da piani passanti per il dato $S_{l k-1}$. E poichè dall' essere $n \geq d \supseteq h$ segue

$$
(a-h)>d-h
$$

ed i piani passanti per un $S_{h_{-1}-1}$ sono $\infty^{l-h}$, il gruppo considerato sarà apolaro a tutte le sezioni fatte in $C^{d}{ }_{n}$ coi piani passanti per l' $S_{h-1}$,

11. Dell' ultima proprietà è utile esporre anche la seguente dimostrazione analitica, la quale deriva immediatamente da una forma notevole che si può dare alla funzione $\Gamma_{h}^{\prime}$. Ricorto a tale seopo che la $\Gamma_{h}^{\prime}$ si costruisce applicando a $d-h+1$ qualunque fra le forme $f_{i}(\lambda)$ lo stesso procedimento che già si è indicato al n." 2 per avere la $\Gamma$, orlando poscia $h$ volte la funzione cosi ottenuta, ed aggiungendo al risultato tutti i termini analoghi. Applicando allora il teorema del sig. Stmphawos a cui si è alluso nel n. 7 (e l'applicazione è lecita, poichè i numeri $h$ ed $m$ di questo Autore qui valgono rispettivamente $d-h$ ed $n$, eppero la condizione $m>k$ è certamente soddisfatta) otteniamo

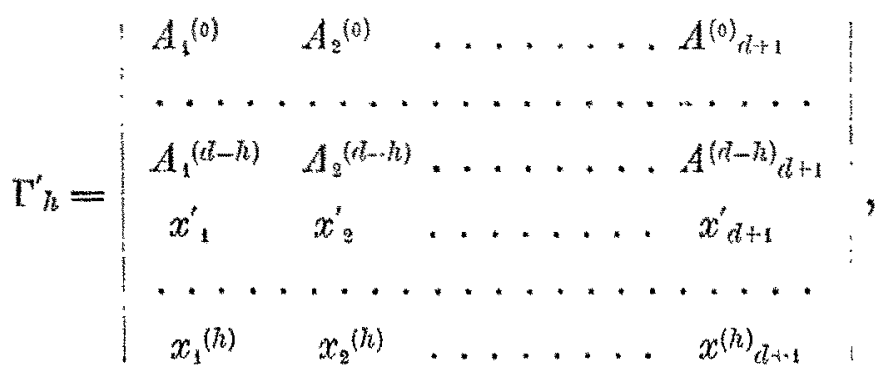

dove in genorale si $\mathrm{e}$ posto

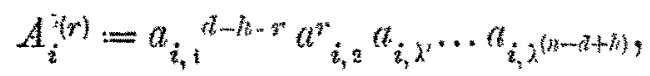

e $\left(x_{1}^{t}, \ldots, x_{d+1}^{\prime}\right), \ldots,\left(x_{1}^{(h)}, \ldots, x^{(h)}{ }_{d+1}\right)$ denotano le coordinate di $h$ punti che determinano lo spazio $S_{h-1}$. Se per semplicita supponiamo poi che questi punti siano quelli che hanno tutte le coordinate nulle, all'infuori rispettivamente della $(d-h+2)^{\mathrm{ma}}, \ldots,(d+1)^{\mathrm{ma}}$, la $\Gamma_{h}^{\prime}$ assume la forma assai semplice

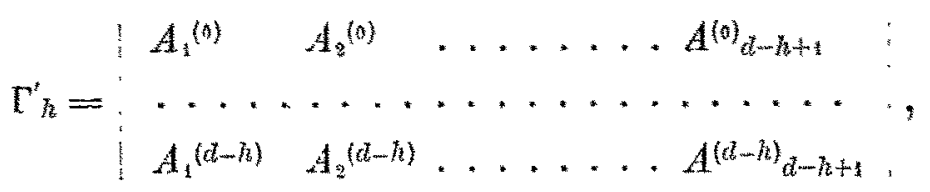


che si può altresl sviluppare come segue:

$$
\Gamma_{h}^{\prime}=\left(a_{1} a_{2}\right) \ldots\left(a_{d-h} a_{d-h+1}\right) a_{1, \lambda} \ldots a_{1, \lambda^{(H-d+h)}} \ldots a_{d-h+1, \lambda} \ldots a_{d-h+1, \lambda^{(h-d+h)}} .
$$

Cio posto, per dimostrare la proprietà di cui si è parlato in principio, indichiamo con $\lambda^{\prime}, \ldots, \lambda^{(n-d+h-1)}$ i parametri di $n-d+h-1$ punti arbitrari di $C^{d_{n}}$ : ad essi, mediante l'equazione $\Gamma_{h}^{\prime}=0$, corrispondono $d-h+1$ punti, che coi primi formano un gruppo dell' involuzione $\left[S_{h-1}\right]_{n-l+h-1}^{n}$. Possiamo dunque dire che un gruppo qualunque di tale involuzione è dato dalle $n$ radici della seguente equazione in $\lambda$ :

$$
\left(\lambda \lambda^{\prime}\right) \ldots\left(\lambda \lambda^{(n-t l+h-1)}\right) \Gamma^{\prime}{ }_{h}=0
$$

dove in $\Gamma_{h}^{\prime}$ in Juogo di $\lambda^{\left(n-d_{+}+h\right)}$ si sia posto $\lambda$. Ora, se per $\Gamma_{h}^{\prime}$ assumiamo l'ultima delle forme date sopra, riesce evidente che il primo membro dell'equazione ora scritta ̀̀ apolare a ciascuna delle forme $f_{1}, \ldots, f_{d \cdots h+1}$, che sono determinate dalle intersezioni di $C_{n}^{d}$ coi piani $x_{1}=0, \ldots, x_{l-n+1}=0$ individuanti l' $S_{h-1}$.

12. La considerazione della (7) permette d'indicare una costruzione assai semplice (e di cui ci serviremo nel $\mathrm{n}^{\circ}{ }^{\circ} 16$ ) dell' involuzione $\left[S_{h-1}\right]^{n}{ }_{n-d+h-1}$. Siano $\lambda^{\prime}, \ldots, \lambda^{(n-(l+h-1)}$ i parametri di $n-d+h-1$ punti qualunque della curva, i quali determinano l'osculante $\left[\lambda^{\prime}, \ldots, \lambda^{\left(n-c_{t}+h-1\right)}\right]_{d-h+1}$. Mantenendo le ipotesi e le notazioni del numero precedente, un piano qualunque passante per l' $S_{h-1}$ è rappresentato dall' equazione

$$
\sum_{i}^{n+h+1} u_{i} x_{i}=0
$$

e taglia quindi la detta osculante nej punti forniti da

$$
\sum_{i}^{(1-n+1} u_{i} a_{i, \lambda^{\prime}} \ldots a_{i, \lambda}{ }^{(j h-d i+h-1)} a_{i, \lambda}{ }^{d \sim h+1}=0 .
$$

Volendo che il detto piano abbia coll'osculante un contatto di ordine $d-\hbar$ nel punto di parametro $\lambda^{(n-l+h)}$, avremo le $d-h+1$ equazioni di condizione

$$
\sum_{i}^{n-h+1} A_{i}^{(0)} u_{i}=0, \ldots, \quad \sum_{i}^{n-l /+1} A_{i}^{(d-h)} u_{i}=0
$$

da cui eliminando le $u_{i}$ si ha appunto, nella forma (7),

$$
\Gamma_{h}^{\prime}=0 \text {. }
$$


Pertanto, dati $n-d+h-1$ punti qualunque sopra $C^{d}{ }_{n}$, $i$ punti, che con essi costituiscono un gruppo dell' involuzione determinata da un dato $S_{h-1}$, hanno per parametri quelli dei punti di contatto dei piani passanti per l' $S_{h-1}$ ed aventi un contatto di ordine $d-h$ coll' osculante mista di ordine $d-h+1$ determinata dai punti considerati.

Se poi ci riferiamo di nuovo al metodo indicato nel $n .^{\circ} 5$, risulta dalle proprietà precedenti che l'involuzione $\left[S_{h-1}\right]^{n}{ }_{n-d+h-1}$ può venir generata come segue. Entro lo spazio $\Sigma_{l}$ si fissi uno spazio lineare qualsivoglia $\Sigma_{d-h}$ di $d-h$ dimensioni, e dicasi $\Sigma_{n-l+h-1}$ il suo spazio conjugato (rispetto ad $F$ od a $\Theta$, secondo che $n$ è pari od impari), che passerà per $\Sigma_{n-d-1}$. Gli $S_{n-1}$ passanti per $\Sigma_{d-h}$ determinano allora sopra $C_{n}^{n}$ un'involuzione di ordine $n$ e di specie $n-\bar{d}+h-1$, la quale, projettata da $\Sigma_{n-l-1}$ in $R_{d}$, diventa l'involuzione $\left[S_{h-1}\right]_{n-a+h-1}^{n}$. L' $S_{h-1}$ di cui si è parlato nei numeri precedenti è l'intersezione di $R_{d}$ con $\Sigma_{n-d+h-1}$.

13. L'involuzione fondamentale, come risulta da teoremi generali ben noti $\left(^{*}\right)$, possiede $(n-d)(d+1)$ punti $(n-d)$-pli, i quali, dovendo $(* *)$ coincidere coi punti $(d+1)$-pli dell' involuzione conjugata, non sono altro che i punti di contatto dei piani aventi con $C^{l_{n}}$ un contatto d'ordine $d$. Per la stessa ragione l'involuzione determinata su $C^{d}{ }_{n}$ da un $S_{h-1}$ possiede $(n-d+h)$ $(d-h+1)$ punti $(n-d+h)$-pli, i quali coincidono coi punti di contatto dei piani passanti per l' $S_{h-1}$, ed aventi colla curva un contatto d'ordine $d-h$.

14. Data un'involuzione di ordine $n$ e di specie $k$, diremo col signor $\mathrm{W}_{\mathrm{EYR}}\left({ }^{* * *}\right)$ che $k$ elementi formano un gruppo neutro della medesima quando essi, insieme con un elemento ulteriore arbitrario, determinano un gruppo dell' involuzione. Volendo trovare il significato geometrico dei gruppi neutri nelle involuzioni sin qui considerate, conviene premettere la ricerca seguente.

Dati sulla curva $r$ punti qualunque $\lambda^{\prime}, \ldots, \lambda^{(r)}$, essi determinano l'osculante $\left[\lambda^{\prime}, \ldots, \lambda^{(r)}\right]_{n-r}$, la quale, quando siano soddisfatte certe condizioni, potrà

(*) Cfr. Buttaglivi, Sulle forme binarie di grado qualumque (Atti della R. Accad. delle Scicnze Fis. e Mat. di Napoli, 1867, vol. III, pag. 20); Fu. WHYr, Ueber Involutionen n-ten Grades and $h$-ter Stufe (Sitzungsb. dell'Acead. di Vienna, tom. 79, 17 aprile 1879, pag. 684). - Una dimostrazione più geometrica di questo e di altri risultati trovasi nel citato lavoro dol sig. Castrinuovo.

(**) Cfr. i lavoni gia ricordati di Stephanos, Brili, Meyer c Castelnuovo.

(***) Loc. cit., \$ 10. Il concetto di gruppo noutro è stato, poi generalizato dal sigunor Castrenufovo nella Momoriat citata. 
giacere in uno o più piani: si tratta di determinare la dimensione e la classe della varietà costituita da questi piani al variare degli $r$ punti sulla $C^{a} t_{n}$. A tal fine osserviamo che, se $u_{1}, \ldots, u_{l+1}$ sono le coordinate di uno di tali piani, esse devono soddisfare, per qualunque valore di $\lambda$, all' equaziono

$$
\alpha_{\lambda^{\prime}} \ldots \alpha_{\lambda}(n) \alpha_{\lambda}^{n+v}=0 \text {. }
$$

Dehbono dunque aver luogo le seguenti relazioni:

$$
\begin{aligned}
& \sum_{i}^{\prime+1} u_{i} a_{i, \lambda} \ldots a_{i, 2, n} a_{i, 1}^{n \cdots \cdots}=0, \\
& \sum_{i}^{\prime+1} n_{i} a_{i, 2} \ldots a_{i, 2} a_{i, 1} a_{i-r-1} a_{i, ?}=0 \\
& \ldots \ldots \ldots \ldots \ldots \\
& \sum_{1}^{n+1} u_{i} a_{i, \lambda} \ldots a_{i, i, k} a_{i, 2}^{n-1}=0 .
\end{aligned}
$$

I $d-(n-r+1)$ rapporti delle $u_{i}$ che, in virtù di esse, restano tuttora arbitrari, dipendono inoltre dagli $r$ parametri $i^{\prime}, \ldots, \lambda^{(*)}$, epperò, volendo che i piani di cui si tratta siano al più $\boldsymbol{\Phi}^{d}$, dovremo supporre

$$
n \geq 2 r^{r}-1 \text {. }
$$

Quando $\sin n=2 r-1$, si possono attribuire alle $u_{i}$ valori quali si vogliano, e le (8) determinano allora, in generale, un sistema unico di valori per le funzioni simmetriche delle $\lambda$ : esiste quindi allora, in generale, una ed una sola osculante mista di ordine $n-r$, situata in un piano dato arbitrariamente.

Se invece $n \supseteq 2 r$, eliminando dalle (8) le $r+1$ funzioni simmetriche omogenee delle $\lambda$, risulta che devono essere nulli tutti i determinanti di ordine $r+1$ tratti dalla matrice

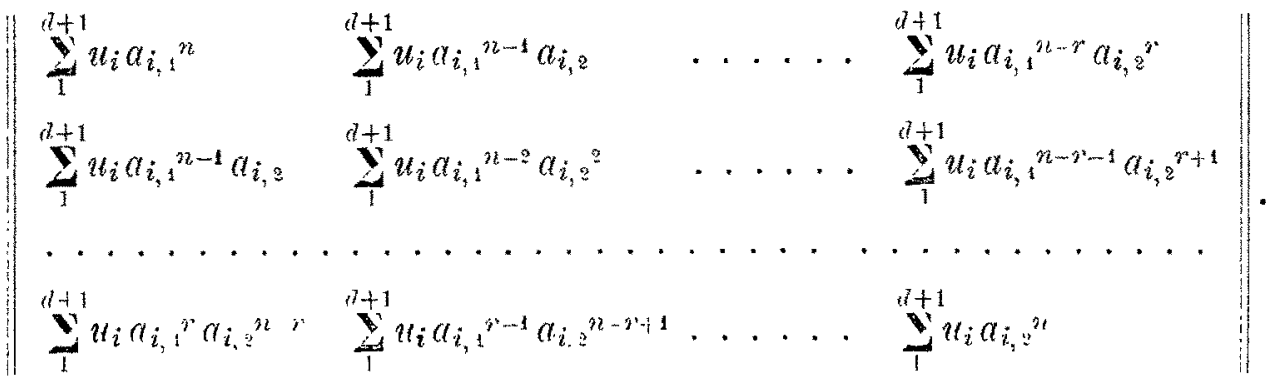


Il problema proposto si risolve allora immediatamente applicando un noto risultato, del quale il sig. Srıhr ha dato recentemente $\left(^{*}\right)$ una dimostrazione assai semplice; si ottiene così:

Quando sia $n \geqslant 2 r>n-d+1$, $i$ piani contenenti le osculanti miste di $r$ punti di una curva $C_{n}^{n}$ formano una varietà $d i d-n+2 r-1$ dimensioni e di classe $\left(\begin{array}{c}n-r+1 \\ r\end{array}\right)$, ciò̀ sono tali che per ogni $S_{d-n+2 r-2}$ ne passano $\left(\begin{array}{c}n-r+1 \\ r\end{array}\right)$.

Se è invece $2 r=n-d+1$ (nel qual caso la prima condizione $n \geq 2 r$ ¿̀ sempre senz'altro soddisfatta), il numero delle osculanti miste di ordine $n-r$ situate in un piano è finito ed uguale a $\left(\begin{array}{c}n-r+1 \\ r\end{array}\right)$.

Ponendo in generale

$$
A_{s}=\sum_{i=1}^{i=d+1} u_{i} a_{i, 1}{ }^{n-s} a_{i, 2}^{s},
$$

i punti, in cui $C^{d}$ è tagliata da un piano arbitrario

$$
\sum_{1}^{d+1} u_{i} x_{i}=0
$$

hanno per parametri le radici dell' equazione

$$
0=\Phi=\sum_{s=0}^{s=n}\left(\begin{array}{l}
n \\
s
\end{array}\right) A_{8} \lambda^{n-s} .
$$

La matrice precedente diviene allora

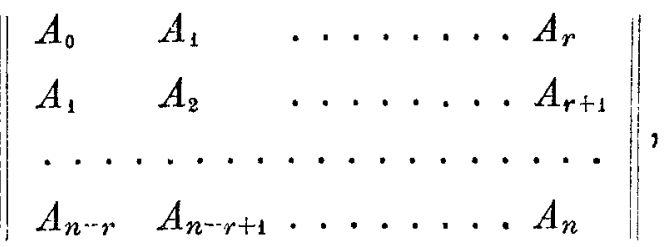

ed all'insieme de'suoi determinanti di ordine $r+1$ il prof. Battaglini (loc. cit., pag. 24) ha dato il nome di plesso cataletticante di ordine $n-2 r$ della

(*) STAHL, Zur Erzougung rler rationalen Raumourven (Math. Ann., Bd. 10, 189?, Anhang, pag. 52).

Annali di Matematica, tomo XXI. 
forma $\Phi$ (cataletticante di $\Phi$ quando $n=2 r$ ), assegnando inoltre il significato geometrico dol suo annullarsi. Dunque:

I piani di cui sopra si ‘ parlato sono quelli che Jianno la proprietà di tagliare $C_{n}^{*}$ in grippi di punti, poi quali is anlo it plesso cataleticante di ordine $n-2 \%$ (rispettivamente il cataletticante).

Per esempio nel piano ordinario $(d=2)$ per $\mathrm{i}$ casi $n=3, r=1 ; n=4$, $r^{*}=2 ; n=5, r=2$, e nello spazio ordinario $(d=3)$ per $\mathrm{i}$ casi $n=4, v=1$; $n=4, r=2 ; n=5, r=2 ; n=6, r=3 ; n=6, r=2 ; n=7, r=3$, si ritrovano lo seguenti proprieta già note.

Per una $C_{3}^{2}$ esistono tre coniche osculanti ridotte ad una retta doppia, c sono le prime osculanti dei tre flessi (Stant, Ueber die Fundamentalinoolutionen, ecc. Giomale di Cresue, Bd. 104, \& 1; Brozorari, Sullu curva del tarz'ordine, coc., loc. cit.).

Una $C^{2}{ }_{4}$ ammette $\infty^{1}$ coniche osculanti degenerate in una rotta doppia: queste rette inviluppano una curva di $3 .^{2}$ classe, o tagliano $C^{*}$ in gruppi armonici di punti; esse non sono poi altro che le tangenti d'inflessione delle primo osculanti (Start, Ueber die rationale ebene Curve vierter Ordnung, Giomale di Creure, Bd. 101, § 3).

In una $C^{2}$ esistono sei osculanti di $3 .^{\circ}$ ordine ridotte ad una retta tripla (Sram, Ueber die Findamentalinolutionen, ecc., loc. cit., $\$ 4$ ).

Per una $C_{3}^{3}$ esistono quattro cubiche osculanti situate in un piano, $c$ sono le prime osculanti dei punti stazionari; i piani delle coniche osculanti, ossia i piani osculatori delle prime osculanti, inviluppano una superficie di $3^{a}$ elasse (la superficie di Sterner di cui $C^{3}{ }_{4}$ è un' assintotica), e tagliano $C^{{ }_{4}}$ in gruppi armonici di punti (Study, loc. cit.; Starr, loc. cit., \$ 2; Berzorarr, Sui combinanti dei sistemi di forme linarie, eco, loc. cit., \$ 5).

Per una $C^{s_{5}}$ i piani contenenti lo osculanti piane di $3 .^{\circ}$ ordine, ossia $i$ piani stazionari delle prime osculanti, formano un fascio gobbo di $6{ }^{a}$ classe (STAIL, loc. cit., $\$ \$ 3^{a}$ e $3^{\circ}$ ).

In una $C^{3}$ i piani delle osculanti piane di $3 .^{\circ}$ ordine, ossia i piani stazionari delle seconde osculanti miste, inviluppano una superficie di $4 .^{\text {a }}$ classe; esistono inoltre dieci osculanti di $4 .{ }^{\circ}$ ordine situate in un piano (STAmr, loc. cit., $\$ 5$ ).

In una $C^{3}$ i piani delle osculanti piane di $4 .{ }^{\circ}$ ordine formano un fascio cobbo di $10 .^{n}$ classe (STurr, loc. cit., $\$ 6$ ).

15. Volendo determinare i gruppi nentri dell'involuzione fondamentalo, si osservi che, in virtù della costruzione esposta al n. ${ }^{\circ} 3$, presi sulla curva 
$n-d-3$ punti qualunque $\lambda^{\prime}, \ldots, \lambda^{(n-a-3)}$, essi insieme con altri due punti $\mu, \nu$ costituiscono un gruppo neutro dell'involuzione, quando l'osculante $\left[\lambda^{\prime}, \ldots\right.$, $\left.\lambda^{(n-d-s)}, \mu, \nu\right]_{d+1}$ è situata in un piano. Ora questa curva è anche l'osculante mista di ordine $d+1$ determinata dai punti $\mu, \nu$ dell'osculante $[\lambda, \ldots$, $\left.i^{\left(n-d_{-3}\right)}\right]_{d+3}$ presa come curva fondamentale; perciò basterà far uso del teorema del numero precedente, ponendo $n=d+3, r=2$. Si ha così:

Dati sulla curva $n-d-3$ punti qualunque $\lambda^{\prime}, \ldots, \lambda^{(n-t l-3)}$, essi fanno parte di $\frac{(d+1)(\lambda+2)}{2}$ gruppi neutri dell' involuzione fondamentale, i quali corrispondono a quelle fra le osculanti miste d'ordine $d+1$ della curva $\left[\lambda^{\prime}, \ldots, \lambda^{(n--d-3)}\right]_{d+3}$, che si trovano in un piano.

16. In modo analogo si determinano $\mathrm{i}$ gruppi neutri di una qualunquo involuzione $\left[S_{h-1}\right]_{n-d+h-1}^{n}$. Dati $n-d+h-3$ punti arbitrari $\lambda^{\prime}, \ldots, \lambda^{(n \ldots l+h+-3)}$ di $C_{n}^{a_{n}}$, essi insieme con altri due punti $\mu, \nu$ formano un gruppo neutro di quell' involuzione, quando l'osculante $\left[\lambda^{\prime}, \ldots, \lambda^{\left(n-a_{4} h_{-3}\right)}, \mu, \nu\right]\left(t-h_{h+1}\right.$ giace in un piano passante per l' $S_{h-1}$ fissato. E poichè tale curva si può anche riguardare come l'osculante mista di ordine $d-h+1$ determinata dai punti $\mu$, $v$ del-

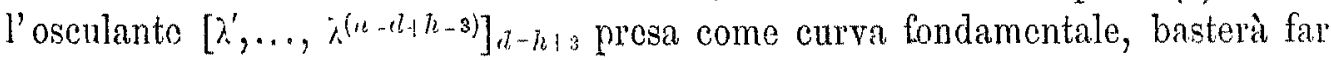
uso del teoremn del n." 14 , ponendo $n=d-h+3, r=2$. Delle due condizioni la trovnle una is sempre soddisfatta, o l'altra diventa

$$
d \equiv h+1
$$

e poichè si ha grià $d \geqslant h$, l'unico caso di ccceziono ò quello in cui $l=h$. Esso è però compreso in quanto si è detto nel principio del n." 14, e si ha pertanto in generale il teorema seguento:

Dato uno spazio lineare qualunque $S_{h}$, e dati sulla curva $n-d+h-3$

munti arbitrari $\lambda^{\prime}, \ldots, \lambda^{(n-a+h-3)}$, essi fanno parte $d i \frac{(d-h+1)(d-h-1-2)}{2}$ gruppi neutri dell'involuzione determinata dal dato $S_{l-1}$ : questi gruppi corrispondono a quelle fra le osculanti miste di ordine $d-h+1$ della curver $\left[\lambda^{\prime}, \ldots, \lambda^{\left(n-d_{+} h_{-3}\right)}\right]_{l \cdots h+3}$, che giacciono in un piano passante per $l$ l' $S_{h-1}$.

Si noti infine che i gruppi neutri dell'involuzione fondamentale e quelli di una qualunque involuzione $\left[S_{h-1}\right]_{n-l+h-1}^{n}$ sono le projezioni in $R_{t l}$ dei gruppi neutri dell'involuzione determinata sopra $C^{n}{ }_{n}$ dagli $S_{n-1}$ passanti rispettivamente por $\Sigma_{l l}$ oppure per il $\Sigma_{l l-h}$ di cui si ò parlato nel n. ${ }^{0} 12$. Gli uni e gli altri si possono dunque costruire nel modo indicato dal sig. CAsTELswovo nel n. ${ }^{0} 11$, loe, cit. 
17. Occupiamoci da ultimo dei gruppi dell'involuzione $\left[S_{h-1}\right]_{n-d+h-1}^{n}$ giacenti in un piano, ed a tale scopo premettiamo le seguenti osservazioni.

Se $n$ è pari, i piani secanti $C^{d}{ }_{n}$ in gruppi di punti apolari a sè stessi inviluppano una quadrica, la cui equazione tangenziale si ottiene uguagliando a zero l'invariante quadratico della forma $\alpha_{2}^{n}$ : questa quadrica, che diremo $Q$, ha per piani tangenti le intersezioni di $R_{d}$ cogli $S_{n-1}$ condotti per $\Sigma_{n-d-1}$ a toccare la quadrica $F$ (vedi $\mathrm{n}^{\circ} 5$ ).

Supponendo invece $n$ dispari, l'inviluppo precedente è indeterminato, e (mantenendo le notazioni del $n^{\circ}{ }^{\circ}$ ) la condizione perchè le sezioni fatte in $C^{d}{ }_{n}$ con due piani differenti

$$
\sum_{1}^{d+1} u_{i} x_{i}=0, \quad \quad \sum_{1}^{d+1} v_{i} x_{i}=0
$$

siano fra loro apolari è

$$
v_{1} \sum_{1}^{u+1} A_{1, i} u_{i}+\cdots+v_{t}+1 \sum_{1}^{d+1} A_{d ! \cdot, i} u_{i}=0
$$

rhe si puì serivere anche come segue:

$$
A_{12} q_{12}+A_{13} q_{13}+\cdots+A_{l l, l+1} q_{l, l l 13}=0,
$$

avendo posto

$$
q_{r s}=u_{r} v_{s}-u_{s} v_{r} .
$$

Le $q_{r s}$ sono le coordinate-assi dell' $S_{d-2}$ comune ai due piani (9), e quando la (11) sia soddisfatta questo $S_{d-2}$ è tale che due piani qualunque del fascio di cui esso è il sostegno tagliano $C^{d}{ }_{n}$ in duo gruppi apolari di punti. La (11) è l'equazione del complesso degli $S_{l-2}$ dotati di tale proprietà.

Considerando la (10), se supponiamo date le coordinate $v_{i}$, essa al variare delle $u_{i}$ rappresenta un punto giacente nel piano $v$, e supponendo date invece le coordinate di questo punto, si potranno da essa ricavare $\mathrm{i}$ valori delle $v_{i}$ nel solo caso in cui non sia nullo il determinante dei loro coefficienti. Fssendo esso gobbo simmetrico e di ordine $d+1$, potrà essere diverso da zero soltanto quando $d$ sia impari. In tal caso la (10) definisce un sistema nullo, che diremo $N$, e nel quale un punto ed un piano fra loro corrispondenti sono tali che tutti i piani passanti per il punto tagliano $C^{a}{ }_{n}$ in gruppi apolari a quello determinato dal piano dato. $\mathrm{E}$ poi anche ovvio il modo con cui questo sistema nullo si può immaginare dedotto da quello che nel $n .^{\circ} 5$ si è chiamato $\Theta$. 
Se invece $d$ è pari, si è dimostrato nel n.. 6 che, fra i gruppi dell' involuzione fondamentale, uno ed uno solo, in generale, ha tutti $i$ suoi punti in un piano, che diremo $\pi$. Se per semplicità si assume $\pi$ come piano avente l'equazione

$$
x_{i+1}=0
$$

sarà

$$
A_{i, d k+1}=0, \ldots, \quad \ddot{A}_{l, d+1}=0,
$$

e la condizione (10) diventa

$$
v_{1} \sum_{i}^{d} A_{4 i} u_{i}+\cdots+v_{d} \sum_{i}^{d} A_{d i} u_{i}=0 .
$$

Da essa risulta che nel caso attuale resta determinato in $\pi$ un sistema nullo, che chiameremo $N_{\pi}$ e nel quale un punto ed un $S_{l-2}$ fra loro corrispondenti sono tali che le sezioni fatte in $C^{d}{ }_{n}$ da due piani qualunque, passanti l'uno per il punto e l'altro per l' $S_{l-2}$, sono apolari.

18. Ciò posto, sia

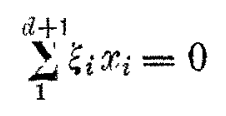

l'equazione di un piano secante $C^{d}{ }_{n}$ in un gruppo dell' involuzione determinata da un $S_{h-1}$ qualunque, di guisa che i parametri dei punti del gruppo stesso saranno le radici dell' equazione

$$
\sum_{i}^{\prime+1} \xi_{i} a^{n}{ }_{i, \lambda}=0 .
$$

Indicando poi con $u_{i, 1}, u_{i, 2}, \ldots, u_{i, l+1}(i=1,2, \ldots, d-h+1)$ le coordinate di $d-h+1$ piani che determinano l' $S_{h-1}$ come loro comune intersezione, le forme

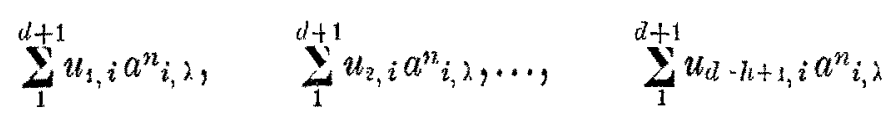

devono essere apolari al primo membro della (13). Scrivendo le condizioni perchè ciò avvenga, si hanno $d-\hbar+1$ relazioni, ed eliminando da esse e dalla (12) le $\xi_{i}$, risulta che le equazioni dello spazio $S_{d-h}$, comune agli $\infty^{h-1}$ piani contenenti gruppi della suddetta involuzione, si nttengono uguagliando a zero $\mathrm{i}$ determinanti di ordine $d-h+2$ tratti dalla matrice 


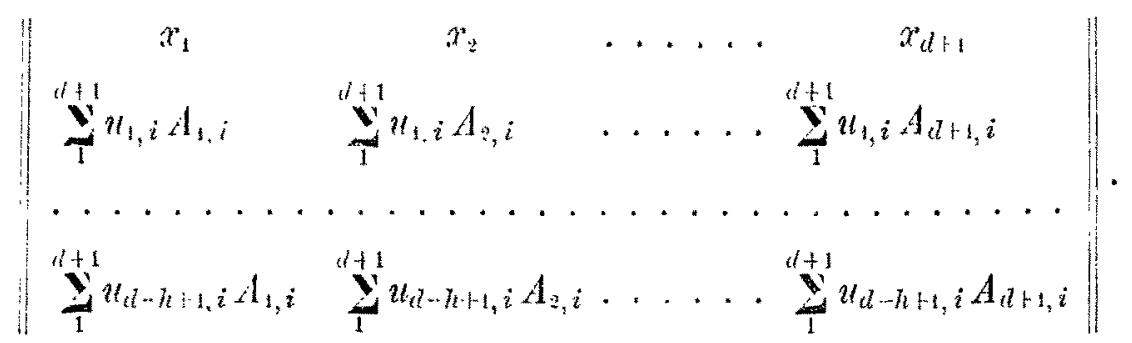

Di qui, ed anche senz'altro dalle cose del numero precedente, risulta:

L'involnzione determinata sopra $C^{d}{ }_{n}$ da uno spazio lineare qualunque $S_{h}$ a possiede $\boldsymbol{c}^{h-1}$ gruppi piani, $i$ cui piani hanno in comune un $S_{t-\hbar}$. Se ni pari, questo $S_{t-h}$ ì lo spazio polare reciproco del dato $S_{h-1}$ rispetto alla quadrica $Q$; se $n$ è dispari e d pure dispari, l' $S_{l-h}$ è lo spazio polare del

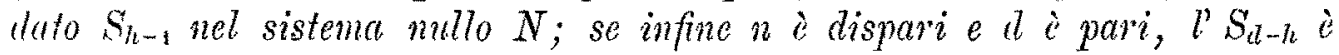
contenuto nel piano $\pi$, ed ì lo spazio polare, nel sistema mullo $N_{\pi}$, della trarria di $\mathrm{S}_{l-1}$ sopra $\pi$.

Colgo quest'occasione per aggiungere al mio lavoro più volte citato Sui combinanti cce. (questi Amali, serie II, tom. 20) un complemento che non mi sombra del tutto sprovvisto d'interesse, o che mi è stato suggerito dallo studio di una Nota del sig. Stephanos (*), della quale ho avuto cognizione soltanto dopo la pubblicazione del lavoro suddetto. Nel $\$ 5$, n. 28 di questo ho dimostrato che i covarianti elemontari delle due forme

$$
5(W w)^{3} W_{\lambda}^{3} w_{\lambda}+2(Q w) Q_{\lambda} w_{\lambda}{ }^{3}, \quad w_{\lambda}^{4}
$$

coincidono, salvo fattori costanti, coi combinanti elementari $W_{\lambda}^{a}$ e $Q_{\lambda}^{i}$, e che percio, in particolare, l'equazione del piano osculatore alla quartica nel suo punto $\lambda$ si ottiene uguagliando a zero il jacobiano delle (1). Ora, per notissime proprieta generali $(* *)$, il discriminante di questo jacobiano si spezza in due fattori, che sono razionali nei coefficienti delle due forme, o le cui espres. sioni, per due forme biquadratiche qualunque, furono date dal sig. Stremanos nella Nota ricordata. ì manifesto che di questi due fattori l'uno, che $i \mathrm{i}$

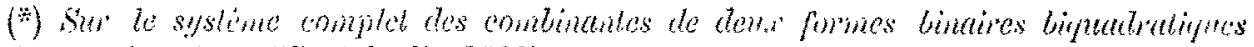
(Comptes remdus, tom. 67,2 lugg lio 1883).

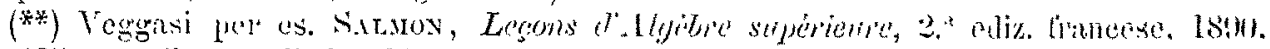

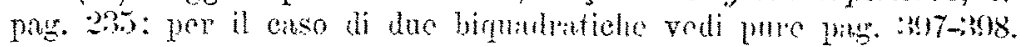


risultante delle (1), rappresenta l'insieme dei quattro piani stazionari della curva, l'altro la sviluppabile osculatrice della medesima (*): il primo, sotto forma leggermente diversa, trovasi già, insieme col suo significato geometrico, alla fine del $\S 3$ del mio lavoro.

Nel caso presente, per quanto ho dimostrato nel loc. cit., n. ${ }^{\circ} 28$, lo forme che il sig. Stephinos chiama $\alpha$ e $\theta$ sono le seguenti:

$$
\alpha_{\lambda}^{6}=-\frac{5}{4} i W_{\lambda}^{6}, \quad \theta_{\lambda}^{2}=-\frac{1}{2} i Q_{\lambda}^{2}
$$

epperò si trova che $\mathrm{i}$ due fattori in cui si decompone il discriminante $\mathrm{di} x \mathrm{r}^{\mathrm{h}}$ sono rispettivamento

$$
\begin{aligned}
& \frac{1}{2^{10} \cdot 3^{2}} i^{4}\left[3^{4} \cdot 5^{2} A^{2}+2^{6} B^{2}-2^{4} \cdot 5 A B-2 \cdot 3 \cdot 5^{5}\left(J J^{\prime}\right)^{4}\right], \\
& \frac{1}{2^{43} \cdot 3^{2}} i^{6}\left[2^{9} \cdot 5^{3} A^{3}+2^{2} \cdot 3 \cdot 5^{2} \cdot 11 A^{2} B-2^{6} \cdot 3^{2} \cdot 5 A B^{2}+2^{8} B^{3}\right. \\
& \left.\quad-3^{3} \cdot 5^{9} A\left(J J^{\prime}\right)^{4}-2 \cdot 3^{2} \cdot 5^{5}\left(J J^{2}\right)^{2}\left(J J^{\prime \prime}\right)^{2}\left(J^{\prime} J^{\prime \prime}\right)^{2}\right] .
\end{aligned}
$$

La quantità che nella (2) è chiusa fra parentesi differisce da quella che nel $\S 3$, loc. cit., ho chiamato $D$ per ciò, che in questa al posto del termine $(W Q)^{\prime}\left(W Q^{\prime}\right)^{2}\left(W Q^{\prime \prime}\right)^{2}$ compare il termine $(J J)^{\prime}$. Ma si può dimostrare facilmente che ha luogo l'identità

$$
3 \cdot 5^{4}\left(J J^{\prime}\right)^{4}-2^{5} B^{2}-2^{2} \cdot 5^{2} A B-2^{3} \cdot 3^{3} \cdot 5(W Q)^{2}\left(W Q^{\prime}\right)^{2}\left(W Q^{\prime \prime}\right)^{2}=0
$$

mediante la quale le due espressioni, salvo un fattore numorico, diventano identiche.

Uguagliando a zero la quantitì che nella (3) sta racchiusa fra parentesi, si ottiene l'equazione della superficie sviluppabile della curva, formata coi combinanti elementari della curva stessa.

Una ricerca analoga potrebbe farsi sulla forma che nel loc. cit. ho chiamato $V_{\lambda}^{6}$, e che, uguagliata a zero, rappresenta il complesso lineare speciale

(*) Un fatto analogo ha luogo por la forma binaria Ldi grado $d(n-d+1)$ nol paramotro $\lambda]$, cho ugragliata a zero rapprosenta il piano osenlatoro nol punto $\lambda$ di una $C^{n z}$ qualunque. Il suo discriminanto si spezza in lue fattori, di cui l'uno rappresenta l'insiemo loi $(d+1)(n-d)$ piani aventi colla curva un contatto d'ordine $f$, o l'altro rappresenta lo spario di $d-1$ dimensioni o d'ordins $(d-1)(n-d+2)$ formato dagli $S_{(i-2}$ osculatori alla curva stessa. 
24 Berzolari: Sulle cure razionali di uno spazio lineare, ece.

avente per asse la tangente alla quartica nel suo punto $\lambda$. Il discriminante di $V_{\lambda^{6}}{ }^{6}$ deve spezzarsi $\left(^{*}\right)$ in due fattori razionali, di cui l' uno rappresenta il complesso di $4 .^{\circ}$ grado costituito dalle rette che si appoggiano in un punto alla curva, e l'altro il complesso di $6 .^{\circ}$ grado formato dalle tangenti alla superficie sviluppabile della stessa. Anche per questo caso si può tenere la via sopra seguita, poichè nel loc. cit., n. ${ }^{\circ} 29$ ho dimostrato che $V_{\lambda}^{6}$ è il jacobiano del fascio di forme biquadratiche determinato sulla curva dai piani passanti per la retta, di cui s'intendono sostituite in $V_{\lambda^{6}}$ le coordinate. Tale fascio si può supporre individuato, per esempio, dal piano che dalla retta data projetta il punto di concorso delle corde principali, e dal piano passante per la retta stessa, c conjugato al precedente rispetto ad una qualunque delle quadriche studiate nol loc. cit., $\$ 4$.

Pavir, 15 novembre 1802.

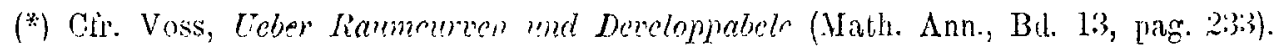

